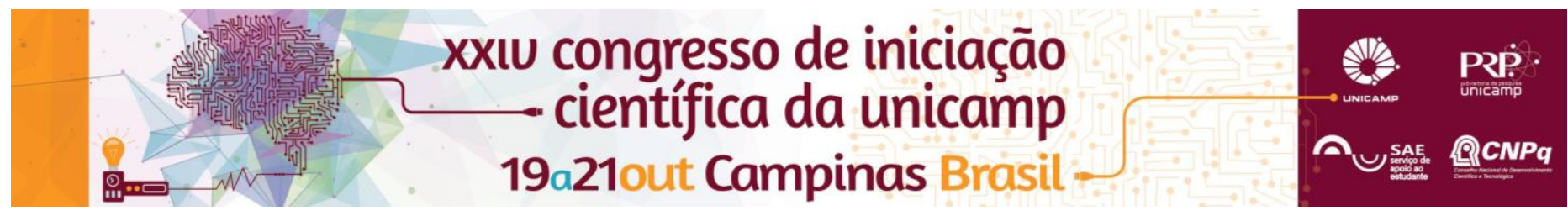

\title{
Design and construction of a microbiome experimental chamber based on Winogradsky's column.
}

\author{
Abans*, Rodrigo A. de O.; Paulino Lima, Ivan G.; Rodrigues, Fabio; Galante, Douglas.
}

\begin{abstract}
In this project, it is presented the development of a simple and eficient Winogradsky's column with climate conditioning and sampling system, to be used as an study model for microbiotic interactions in a simulated extraterrestial environment, such as of Mars. This chamber allows a variety of experiments in the field of astrobiology.
\end{abstract}

\section{Key words: \\ Winogradsky; atrobiology; Mars .}

\section{Introduction}

Recently, there have been significant discoveries about Mars that have increased our interest in it and in the possibilities of more advanced robotic and even manned missions to that planet. Thereby, many questions about Mars's inhabitability, search of life forms and life support systems for long duration manned missions are arising. Within this line of thought, a Winogradsky's column shows potential as a tool to investigate such problems.

The first concept for the column was developed by Sergei Winograsky ${ }^{1}$ (apud QUINN et al., 2014) for the study of microcommunities' stratification under oxygen, light and sulfur gradients. Since then, the columns have been excellent models to develop microecology studies under environmental changes (RUNDELL et al., 2014) and have even found applications in space engineering, such as recycle systems like MELiSSA (HENDRICKX et al, 2005).

Thus, inspired in Mars Ecopoiesis Test Bed (TODD [Org.], 2006), it was developed of a Winogradsky-based column as an self-contained ecosystem, analog to what could exist in other planets, as a model to study biotic interactions in a extraterrestrial environment.

\section{Results and Discussion}

The Winogradsky column was designed to be simple, cost-effective, easy to manipulate and, at the same time, robust and scientifically trustworthy, with the characteristics that follow:

With the exception of the tubing and cover, everything else is not permanently attachable, allowing easy disassemble and substitution of parts, as needed. Most of the parts of the chamber are made of transparent acrylic (laser cut), hence visible changes inside the column can be easily monitored, if that's the objective. One piece, which seals and binds the middle of the column, was made from teflon for its easy craft and low chemical reactivity.

The column was made to withstand small external and internal pressure variations, but its design allows easy modification depending of the experiment. Likewise, the column is very gas tight and totally water sealed, allowing pre-assemble in an sterile environment without contamination concern during transportation. Its cover was made with PDMS (Sylgard 184, Dow Corning), a polymer with great strength and good transparency on the UV range $(>230 \mathrm{~nm})$, allowing different types of irradiation experiments, even on shorter wavelengths.

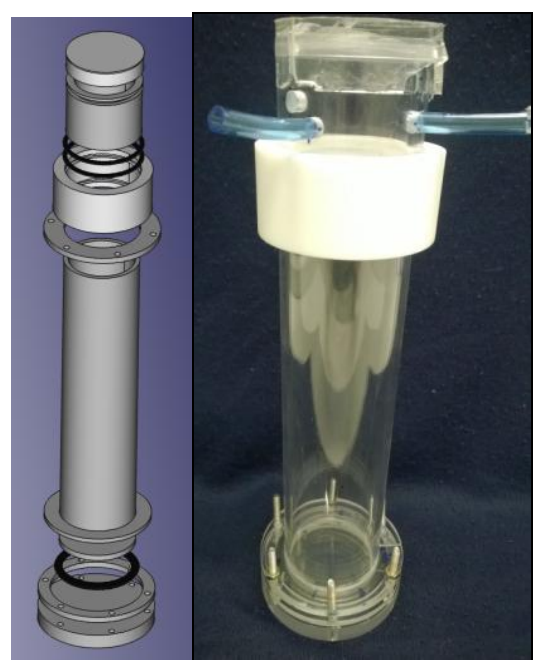

Image 1. left: design of all pieces and orientation of assemble; right: column setup accordingly to design.

\section{Conclusions}

This design of an adapted Winogradsky's column was a success and it will soon be applied on the sequel of this project to study microbiological interactions under Marslike conditions. It is expected that this experiment will be a stepping stone on the development of an experiment to be launched to the stratosphere with a balloon, and even as a small payload for satellites, as a part of the goals of the research group in astrobiology of LNLS.

\section{Acknowledgement}

I thank everyone of CNPEM and from other institutions that gave me your support and patience to hear my questions, especially the machine shop (OFI/CNPEM). I thank the financial support of CNPq through the PIBIC.

-HENDRICKX, L. et al. Microbial ecology of the closed artificial ecosystem MELiSSA (Micro-Ecological Life Support System Alternative): reinventing and compartmentalizing the Earth's food and oxygen regeneration system for long haul space exploration missions. Research in Microbiology: vol. 157, pp. 7786, Dez. 2005

-QUINN, R. A. et al. A Winogradsky-based culture system shows an association betweeen microbial fermentation and cystic fibrosis exacerbation. The ISME Journal: vol. 09, pp. 1024-1038, Dez. 2014.

-RUNDELL, E. A. et al. 16S rRNA gene survey of microbial communities in Winogradsky columns. Plos One: vol. 90, no. 08, Ago. 2014.

Winogradsky, S. N. O roli mikrobov v krugovorote zhizni [On the role of microbes in the cycle of life]. Arkhiv Biol Nauk: vol. 07, pp. 01-27, 1897.

-TODD, P. (Org.). Phase II Final Progress Report: robotic lunar ecopoiesis tes bed. 2006. 108f. Greenville, Universities Space Research Association, 2006. 